\title{
ADAPTIVE TRACKING CONTROL FOR TWIN ROTOR MULTIPLE-INPUT MULTIPLE-OUTPUT BASED ON ISS STABILIZATION
}

\author{
Nguyen Van Chi $^{{ }^{*}}$ \\ ${ }^{I}$ Thai Nguyen University of Technology, 3/2 Street Tich Luong, Thai Nguyen \\ "Email:ngchi@tnut.edu.vn
}

Received: 30 July 2015; Accepted for publication: 03 August 2016

\begin{abstract}
This paper proposes the angle tracking control method for Twin rotor multi-input multipleoutput (TRMS) using the input-to-state stability theory (ISS) for nonlinear systems. To apply this theory, the model of TRMS is rewritten by an Euler-Lagrange forced model with uncertain parameters and input disturbances. The uncertain parameters are the potential energies depended on the mass of TRMS'parts and the input disturbances are the considered friction force, flat cable force, and effects of the speed of the main rotor on the horizontal movement and the speed of tail rotor to the vertical movements. Using modificated model of TRMS, we designed the adaptive controller for angle ISS stabilization to attenuate the influences of uncertain parameters and input disturbances to the angles of TRMS. The robustness of the closed system is shown by the the stabilization of the angles with the yaw and pitch external disturbances, the simulation and experimental results help to proof the rightness of proposed method.
\end{abstract}

Keywords: adaptive tracking, Twin rotor multiple-input multiple output, ISS stabilization, robust adaptive feedback control, uncertain systems, Euler-Lagrange forced model.

\section{INTRODUCTION}

The Twin rotor multi-input multiple-output (TRMS) system was manufactured by Feedback Instrument as shown in Fig. 1. TRMS is a fully actuated mechanical system with two links, a horizontal link connected to the tower through a pivot and another link is perpendicular to the horizontal link connected through a rotational joint with propellers attached at both ends. TRMS is a nonlinear system including the vertical and horizontal movements which is driven by the propulsive forces due to the main rotor and the horizontal tail rotor respectively, the propulsive forces can be changed by the voltages applied to the DC motors [1]. The yaw and the pitch angles are measured by tachometers. The TRMS model is used to test the control law in the laboratory, the important application of the TRMS model is experiments of control problems for the helicopter [2] because it is an experimental set-up that resembles with the helicopter model

The angle stabilization control problem for TRMS is difficult because of the dynamic characteristics of TRMS, high nonlinear systems with high coupling between the horizontal 
motion and vertical motion, the friction moment, the cable moment and gyro moment influence to the propulsive moments as input disturbances which can not be modeled exactly in the practice. As the rotor speeds are varying, high amount of cross coupling creeps into the system which no longer keeps systems flat.

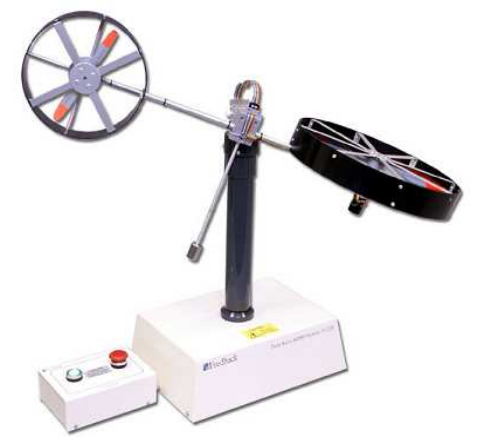

Figure 1. The TRMS setup in the Instrument and Control Lab of Thai Nguyen University of Technology.

In addition, there is very difficult to get the exact model of TRMS because many physical parameters can not be measured exactly, the parameters supplied by the manufactory are changed by the time when TRMS is used in practice, especially the inertia constant, friction coefficient, viscosity constant, the sign function in the propulsive forces that influence the performance of system and the angle tracking errors.

Many tracking control strategies applied to the TRMS have been investigated during the last decade. In the references [3],[4] the authors presented the using controller PID and PID with derivative filter coefficient. With uncertain parameters and external disturbance, the closed loop driven by PID controller keeps the undesirable responses including large overshoot, oscillations and large setting time. The references [5],[6] concerned the control for TRMS using fuzzy logic controller and PID controller. By using the fuzzy controller, the performance of system is better, so to capture the uncertainties there are so many times to try and try the fuzzy laws, the membership functions. Reference [7] considerd the using the LQR controller based on the linearization model of TRMS in the hover mode and an optimal state feedback controller based on linear quadratic regulator (LQR) technique has been applied for TRMS. The difficulty to apply for tracking problems and how to choose matrices $\mathrm{Q}$ and $\mathrm{R}$ are the disadvantages of this method. The reference [8] refered to TRMS controlled by the terminal sliding mode control that keeps the system to be stable to disturbance in pitch and yaw, this method used the linearization and analysis of zero dynamics to control TRMS at the operation point, the sliding mode controller responds quickly in attenuating the disturbances. Using artificial neural networks and genetic algorithms, the adaptive model inversion control approach was presented in [9],[10].

This paper applied the input-to-state stability theory (ISS) to design the adaptive controller for stabilizating yaw and pitch angles for TRMS in the presence of all disturbances and uncertain parameters for the angle tracking problem. Firstly, the mathematical model of TRMS is rewritten in Euler-Lagrange forced model with uncertain parameters and input disturbances that are respectively the energies depended on the mass of TRMS'parts and the friction force, the flat cable force, the effects of the speed of the main rotor on the horizontal movement and the speed of tail rotor to the vertical movements. The output of the controller are the rotation speeds of two DC motors which are the desired set points of the inner control loop by the input voltages applied to the DC motors. By choosing appropriately adaptive controller parameters, the effects of the input disturbances to the yaw and pitch angles will be attenuated. 
The paper is organized as follows. Next section deals with the rewriting the model of the TRMS , followed by the design of adaptive controller for TRMS based on ISS stabilization, the proof of robustness of closed loop are given in this section. Section 4 deals with the results obtained from simulations and experiments, and last section consists of conclusions.

\section{EULER-LAGRANGE FORCED MODEL OF THE TRMS}

Accurate modeling of the system is very important for developing the control law for TRMS. Authors in the [11] presented the dynamic model of TRMS using the Lagrangian method which is took all the effective forces into account. Now, we consider (see notation in Fig. 2):

$\alpha_{h}, \alpha_{v}$ : Horizontal and vertical angles (measured outputs),

$\omega_{h}, \omega_{v}$ : Rotational speeds of tail rotor and main rotor.

$k_{f h n}, k_{f v p}, k_{f v n}, J_{1}, J_{2}, J_{3}, m T_{1}, l_{T_{1}}, m T_{2}, l_{T_{2}}, g, h, l_{t}, l_{m}$ and $k_{m}, k_{g}, k_{f h p}$, are the physical parameters and defined parameters of the TRMS listed in the appendix of this paper.

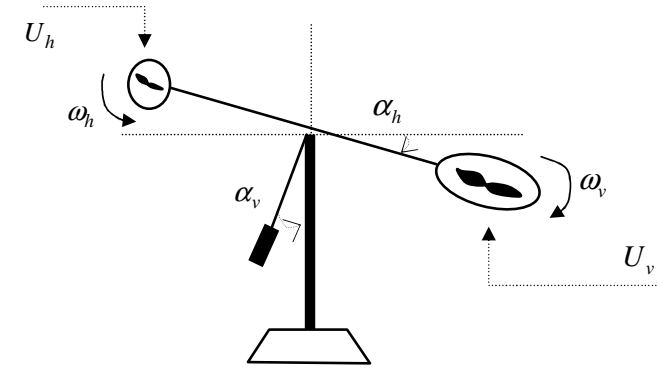

Figure 2. The denotations of TRMS used in the model formulations.

From [11], the model of TRMS is rewritten in Euler- Lagrange forced model as follow

$$
M(\underline{\alpha}) \underline{\ddot{\alpha}}+C(\underline{\alpha}, \underline{\dot{\alpha}}) \underline{\dot{\alpha}}+G(\underline{\alpha})=\mathfrak{I}
$$

where $\underline{\alpha}=\left[\begin{array}{ll}\alpha_{h} & \alpha_{v}\end{array}\right]^{T}$ is state vector, the matrixes $M(\underline{\alpha}) \in R^{2 \times 2}, C(\underline{\alpha}, \underline{\dot{\alpha}}) \in R^{2 \times 2}, G(\underline{\alpha}) \in R^{2 \times 1}$ are the system matrices defined as

$$
\begin{gathered}
M(\underline{\alpha})=\left[\begin{array}{c:c}
J_{1} \cos ^{2} \alpha_{v}+J_{2} \sin ^{2} \alpha_{v} & h\left(m_{T_{1}} l_{T_{1}} \sin \alpha_{v}\right. \\
+h^{2}\left(m_{T_{1}}+m_{T_{2}}\right)+J_{3} & \left.-m_{T_{2}} l_{T_{2}} \cos \alpha_{v}\right) \\
\hdashline h\left(m_{T_{1}} l_{T_{1}} \sin \alpha_{v}-m_{T_{2}} l_{T_{2}} \cos \alpha_{v}\right) & J_{1}+J_{2}
\end{array}\right] \\
C(\underline{\alpha}, \underline{\dot{\alpha}})=\left[\begin{array}{c:c}
2\left(J_{2}-J_{1}\right) \sin \alpha_{v} \cos \alpha_{v} \dot{\alpha}_{v} & h\left(m_{T_{1}} l_{T_{1}} \cos \alpha_{v}\right. \\
\hdashline\left(J_{1}-J_{2}\right) \sin \alpha_{v} \cos \alpha_{v} \dot{\alpha}_{h} & 0 \\
0 & 0
\end{array}\right] \\
C(\underline{\alpha})=\left[\begin{array}{c}
\left.l_{T_{2}} \sin \alpha_{v}\right) \dot{\alpha}_{v} \\
\hdashline g\left(m_{T_{1}} l_{T_{1}} \cos \alpha_{v}+m_{T_{2}} l_{T_{2}} \sin \alpha_{v}\right)
\end{array}\right]
\end{gathered}
$$

and $\mathfrak{I}=\left[\begin{array}{ll}\sum_{i} \tau_{i h} & \sum_{i} \tau_{i v}\end{array}\right]^{T} \in R^{2 \times 1}$ with the elements $\sum_{i} \tau_{i h}, \sum_{i} \tau_{i v}$ are the sum of applied 
torques in the horizontal and vertical movements and can be summarized as

$$
\sum_{i} \tau_{\text {ih }}=\tau_{\text {proh }}-\tau_{\text {frich }}-\tau_{\text {cable }}\left(\alpha_{h}\right)+\tau_{h v}
$$

$\tau_{\text {proph }}=l_{t} F_{h}\left(\omega_{h}\right) \cos \alpha_{v}$ is the propulsive force due to the tail rotor, $\tau_{\text {frich }}$ implies the torque of the friction force, $\tau_{\text {cable }}\left(\alpha_{h}\right)$ refers to the torque of the flat cable force, the last term $\tau_{h v}=k_{m} \dot{\omega}_{v} \cos \alpha_{v}$ of (5) represents the effect of the main propeller speed on horizontal movement.

$$
\sum_{i} \tau_{i v}=\tau_{\text {prov }}-\tau_{\text {fricv }}+\tau_{v h}+\tau_{\text {gyro }}
$$

$\tau_{\text {propv }}=l_{m} F_{v}\left(\omega_{v}\right)$ represents the torque of propulsive force due to the main rotor, $\tau_{\text {fricv }}$ is the torque of the friction force, $\tau_{v h}=k_{t} \dot{\omega}_{h}$ denotes the effect of the tail propeller speed on vertical plane movement of the beam, $\tau_{\text {gyro }}=k_{g} F_{v}\left(\omega_{v}\right) \dot{\omega}_{h} \cos \alpha_{v}$ refers to the torque of the gyroscopic effect.

The functions $F_{h}\left(\omega_{h}\right), F_{v}\left(\omega_{v}\right)$ are given by the following equations

$$
\begin{aligned}
& F_{h}\left(\omega_{h}\right)= \begin{cases}k_{f h p}\left|\omega_{h}\right| \omega_{h} & \omega_{h} \geq 0 \\
k_{f h n}\left|\omega_{h}\right| \omega_{h} & \omega_{h}<0\end{cases} \\
& F_{v}\left(\omega_{v}\right)= \begin{cases}k_{f v p}\left|\omega_{v}\right| \omega_{v} & \omega_{v} \geq 0 \\
k_{f v n}\left|\omega_{v}\right| \omega_{v} & \omega_{v}<0\end{cases}
\end{aligned}
$$

where $\omega_{h}, \omega_{v}$ are the rotational speed of tail and main rotor, respectively.

We rewrite the matrix $M(\underline{\alpha})$ of the (1) as below

$$
M_{p}(\underline{\alpha}) \underline{\ddot{\alpha}}+C(\underline{\alpha}, \underline{\dot{\alpha}}) \underline{\dot{\alpha}}+G(\underline{\alpha})=\mathfrak{I}-\left[\begin{array}{l}
h\left(m_{T_{1}} l_{T_{1}} \sin \alpha_{v}-m_{T_{2}} l_{T_{2}} \cos \alpha_{v}\right) \ddot{\alpha}_{v} \\
\hdashline h\left(m_{T_{1}} l_{T_{1}} \sin \alpha_{v}-m_{T_{2}} l_{T_{2}} \cos \alpha_{v}\right) \ddot{\alpha}_{h}
\end{array}\right]
$$

where the matrix

$$
M_{p}(\underline{\alpha})=\left[\begin{array}{c:c}
J_{1} \cos ^{2} \alpha_{v}+J_{2} \sin ^{2} \alpha_{v} & 0 \\
+h^{2}\left(m_{T_{1}}+m_{T_{2}}\right)+J_{3} & 0 \\
\hdashline 0 & J_{1}+J_{2}
\end{array}\right]
$$

is defined positive matrix.

The model of TRMS now becomes

$$
M_{p}(\underline{\alpha}) \underline{\ddot{\alpha}}+C(\underline{\alpha}, \underline{\dot{\alpha}}) \dot{\alpha}+G(\underline{\alpha})=\mathfrak{I}_{\text {prop }}+\tau_{d}
$$

where $\mathfrak{I}_{\text {prop }}=\left[\begin{array}{ll}\mathfrak{J}_{\text {proph }} & \mathfrak{I}_{\text {propv }}\end{array}\right]^{T}$ is input torque vector applied to the TRMS, $\tau_{d}$ is considered the input disturbance torque vector

$$
\tau_{d}=\left[\begin{array}{c}
-\tau_{\text {frich }}-\tau_{\text {cable }}\left(\alpha_{h}\right)+k_{m} \dot{\omega}_{v} \cos \alpha_{v} \\
-h\left(m_{T_{1}} l_{T_{1}} \sin \alpha_{v}-m_{T_{2}} l_{T_{2}} \cos \alpha_{v}\right) \ddot{\alpha}_{v} \\
-\tau_{\text {fricv }}+k_{t} \dot{\omega}_{h}+\tau_{\text {gyro }}-h\left(m_{T_{1}} l_{T_{1}} \sin \alpha_{v}-m_{T_{2}} l_{T_{2}} \cos \alpha_{v}\right) \ddot{\alpha}_{h}
\end{array}\right]
$$

From the matrix $M_{p}(\underline{\alpha})$ we conclude that the dynamics of angles $\alpha_{h}, \alpha_{v}$ are strongly effected by $J_{1}, J_{2}, J_{3}$ but in the practice, we can not get the parameters $J_{1}, J_{2}, J_{3}$ exactly, so we assume it 
to be unknown constant parameters. This unknown parameters is adapted in the performance to keep the tracking errors of the system. In this paper we consider the constant uncertain vector defined by

$$
\underline{\theta}=\left[\begin{array}{lll}
\theta_{1} & \theta_{2} & \theta_{3}
\end{array}\right]^{T}=\left[\begin{array}{lll}
J_{1} & J_{2} & J_{3}
\end{array}\right]^{T}
$$

Therefore, the Euler- Lagrange forced model of the TRMS perturbed by input disturbance and contained constant uncertain parameters is represented by

$$
\begin{aligned}
& M_{p}(\underline{\alpha}, \underline{\theta}) \underline{\ddot{\alpha}}+C(\underline{\alpha}, \underline{\dot{\alpha}}, \underline{\theta}) \underline{\dot{\alpha}}+G(\underline{\alpha}, \underline{\theta}) \\
& =\underbrace{\mathfrak{I}_{\text {prop }}}_{\text {input torque vector }}+\underbrace{\tau_{d}(\underline{\theta})}_{\text {disturbance torque inputs }}
\end{aligned}
$$

The equation (14) can be expressed by affine with the constant uncertain vector as follow

$$
M_{p}(\underline{\alpha}, \underline{\theta}) \underline{\ddot{\alpha}}+C(\underline{\alpha}, \underline{\dot{\alpha}}, \underline{\theta}) \underline{\dot{\alpha}}+G(\underline{\alpha}, \underline{\theta})=F_{0}(\underline{\alpha}, \underline{\dot{\alpha}}, \underline{\ddot{\alpha}})+F_{1}(\underline{\alpha}, \underline{\dot{\alpha}}, \underline{\ddot{\alpha}}) \underline{\theta}
$$

where the matrix $F_{1}(\underline{\alpha}, \underline{\dot{\alpha}}, \underline{\ddot{\alpha}})$ is written

$$
F_{1}(\underline{\alpha}, \underline{\dot{\alpha}}, \underline{\ddot{\alpha}})=\left[\begin{array}{c:c:c}
f_{11} & f_{12} & f_{13} \\
\hdashline f_{21} & f_{22} & f_{23}
\end{array}\right]
$$

with the elements of the $F_{1}(\underline{\alpha}, \underline{\dot{\alpha}}, \underline{\ddot{\alpha}})$ are formulated as follow:

$$
\begin{aligned}
& f_{11}=\cos ^{2} \alpha_{v} \ddot{\alpha}_{h}-2 \dot{\alpha}_{v} \dot{\alpha}_{h} \sin \alpha_{v} \cos \alpha_{v}, f_{12}=\sin ^{2} \alpha_{v} \ddot{\alpha}_{h}+2 \dot{\alpha}_{v} \dot{\alpha}_{h} \sin \alpha_{v} \cos \alpha_{v} \\
& f_{13}=\ddot{\alpha}_{h}, f_{21}=\ddot{\alpha}_{v}+\dot{\alpha}_{h}^{2} \sin \alpha_{v} \cos \alpha_{v} \\
& f_{22}=\ddot{\alpha}_{v}-\dot{\alpha}_{h}^{2} \sin \alpha_{v} \cos \alpha_{v}, f_{23}=0
\end{aligned}
$$

The equations (12),(13),(14),(15) and (17) represent the TRMS in Euler- Lagrange forced model perturbed by input disturbance and contained constant uncertain parameters. Using these equations, next we proposed the design of adaptive controller based on ISS stabilization to drive the angles of the TRMS tracking to the desired angles.

\section{ADAPTIVE CONTROLLER FOR TRMS BASED ON ISS STABILIZATION}

The dynamic of the system (14) depends on the uncertain parameters and input disturbances which are inputs considered the exogenous signals. The closed systems is asymptotic stability of the original if:

$$
\lim _{t \rightarrow \infty}\left(\underline{\alpha}\left(t, \underline{\tau}_{d}\right)-\underline{\alpha}_{r}\left(t, \underline{\tau}_{d}\right)\right)=0 \quad \forall \underline{\tau}_{d}(t)
$$

and uniform asymptotic stability of the original if:

$$
\left|\underline{\alpha}\left(t_{1}, \underline{\tau}_{d}\right)-\underline{\alpha}_{r}\left(t_{1}, \underline{\tau}_{d}\right)\right|<\left|\underline{\alpha}\left(t_{2}, \underline{\tau}_{d}\right)-\underline{\alpha}_{r}\left(t_{2}, \underline{\tau}_{d}\right)\right| \quad \forall t_{1}>t_{2} \text { and } \forall \underline{\tau}_{d}(t)
$$

There is difficult to drive the closed system meeting the performance criteria (18) or (19), Sontag in 12[12] proposed the extra stabilization definition that is Input to State Stability (ISS). The closed system called ISS stabilization if there is a attractor $\Xi$ of the original such that all trajectories of vector $\left(\underline{\alpha}\left(t, \underline{\tau}_{d}\right)-\underline{\alpha}_{r}\left(t, \underline{\tau}_{d}\right)\right)$ always tend to $\Xi$. For the tracking control problem of model (14), the input disturbance vector is assumed to be bounded:

$$
\delta=\sup _{t}\left|\tau_{d}(t)\right|
$$


Based on [13], in [14, 15] and [16] we proposed an adaptive controller for the input perturbed uncertain systems to tracking control in the sense that the tracking error has to be bounded for all $t \geq 0$ and asymptotically convergence to the origin. Using this proposed controller, we apply to the TRMS with model (14) to calculate the input torque vector applied to the TRMS, the adaptive feedback linear controller is

$$
\left\{\begin{array}{l}
\frac{d \hat{\theta}}{d t}=(\Phi F)^{T} P \underline{x} \\
\mathfrak{J}_{\text {prop }}=D_{P}(\underline{\alpha}, \hat{\theta})\left[\ddot{\alpha}_{r}+K_{1} \underline{e}+K_{2} \underline{\dot{e}}\right]+C(\underline{\alpha}, \underline{\dot{\alpha}}, \underline{\hat{\theta}}) \underline{\dot{\alpha}}+G(\underline{\alpha}, \underline{\hat{\theta}})
\end{array}\right.
$$

where $\underline{e}=\underline{\alpha}-\underline{\alpha}_{r}$ is the tracking errors, $\underline{\alpha}_{r}$ is any desired angles, the $4 \times 2$ matrix $\Phi$ is defined by:

$$
\Phi=\left(\begin{array}{c}
\Theta \\
-M_{p}^{-1}(\underline{\alpha}, \underline{\hat{\theta}})
\end{array}\right)
$$

in which $\Theta$ is the $2 \times 2$ zeros matrix, $K_{1}, K_{2}$ are any two selected $2 \times 2$ matrices such that $4 \times 4$ matrix:

$$
A=\left(\begin{array}{c:c}
\Theta & I \\
\hdashline-K_{1} & -K_{2}
\end{array}\right)
$$

with the $2 \times 2$ identity matrix I, will be Hurwitz, and the symmetric positive definite $4 \times 4$ matrix $P$ is the solution of the Lyapunov equation:

$$
\frac{1}{2}\left(A^{T} P+P A\right)=-Q
$$

where $\mathrm{Q}$ is also an arbitrarily chosen symmetric positive definite $4 \times 4$ matrix.

The adaptive feedback controller (21) given above always drives the tracking errors $\underline{x}=\operatorname{col}(\underline{e}, \underline{\dot{e}})$ of the closed loop system depicted in Fig. 3 asymptotically to the neighborhood $\mathbb{Z}$ of the origin defined by:

$$
\Xi=\left\{\underline{x} \in R^{4}|| \underline{x} \mid \leq \frac{\|P \Phi\| \delta}{\lambda_{\text {min }}(Q)}\right\}
$$

Since the feedback linearization controller (21) contains in it some freely selected parameters such as two matrices $K_{1}, K_{2}$ and the symmetric positive definite matrix P, the robust tracking performance defined in the equation (25) above of the closed loop system depicted in Fig. 3 could be evidently improved further, if these parameters have been suitably chosen. And next, we will present a methodology to determine matrices $K_{1}, K_{2}, P$ for adaptive linearization controller (21) so that the tracking behavior of the obtained closed loop system satisfies any desired arbitrarily small attractor $\Xi$. 


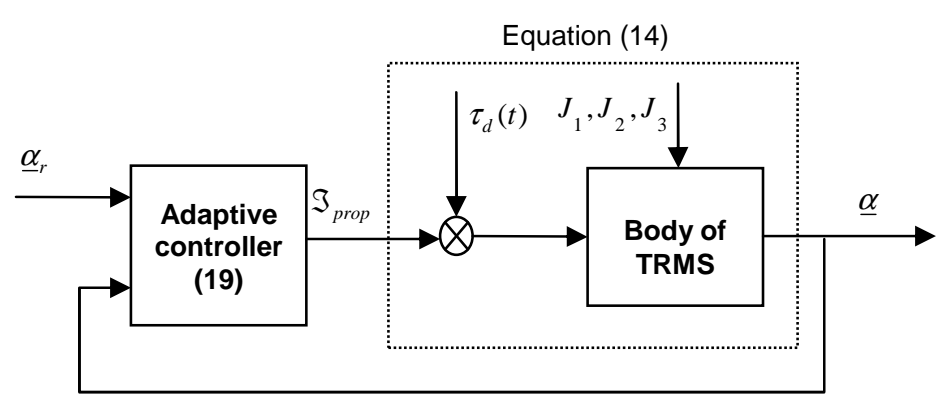

Figure 3. Structure of the closed loop system obtained by using the adaptive controller (21).

Also according to the suggestion of [13], both matrices $K_{1}, K_{2}$ of the adaptive controller (21) could be chosen diagonally:

$$
K_{1}=\operatorname{diag}\left(k_{1 i}\right), K_{2}=\operatorname{diag}\left(k_{2 i}\right), i=1,2, \ldots, n
$$

and appropriately the matrix $\mathrm{Q}$ of the form:

$$
Q=\left(\begin{array}{c:c}
K_{1}^{2} & \Theta \\
\hdashline \Theta & K_{2}^{2}-K_{1}
\end{array}\right)=\left(\begin{array}{c:c}
\operatorname{diag}\left(k_{1 i}^{2}\right) & \Theta \\
\hdashline \Theta & \operatorname{diag}\left(k_{2 i}^{2}-k_{1 i}\right)
\end{array}\right)
$$

In this circumstance the matrix $\mathrm{A}$ is Hurwitz if and only if $k_{1 i}>0, k_{2 i}^{2}>k_{1 i}$ for all $i=1,2, \ldots, n$ and the Lyapunov equation has the following unique solution:

$$
P=\left(\begin{array}{c:c}
2 K_{1} K_{2} & K_{1} \\
\hdashline K_{1} & K_{2}
\end{array}\right)
$$

which is obviously symmetric and positive definite. Moreover, it is easily to recognize from the equation (25), that the measure of $\Xi$ defined as follows:

$$
\Omega(\Xi)=\max _{\underline{x}, \underline{y}}|\underline{x}-\underline{y}| \text { for all } \underline{x}, \underline{y} \in \Phi
$$

is an intuitive value to appreciate the robustness of the closed loop system. The smaller $\Omega(\Xi)$ is, the better robustness of the system is, therefore the closed system which contains the model (14) and adaptive controller (21) is called ISS stabilization.

Theorem 1. For the system (14) perturbed by input disturbance and contained constant uncertain parameters and any given $\beta>0$ always exits two matrices $K_{1}, K_{2}$ such that the proposed feedback dynamic controller Error! Reference source not found. satisfies the desired robustness:

$$
\Omega(\Xi) \leq \beta
$$

Proof: Chosen $K_{1}, K_{2}$ diagonally with:

$$
K_{1}=\operatorname{diag}(k), k>1 \text { and } K_{2}=\operatorname{diag}(a k), a>\sqrt{2}
$$

as well as $\mathrm{Q}$ from the structure (27), then there are obtained: 


$$
\|P \Phi\|=\left\|\left(\begin{array}{c:c}
2 K_{1} K_{2} & K_{1} \\
\hdashline K_{1} & K_{2}
\end{array}\right)\left(\begin{array}{c}
\Theta \\
\hdashline \hat{M}_{p}^{-1}
\end{array}\right)\right\|=\left\|\left(\begin{array}{c}
K_{1} \hat{M}_{p}^{-1} \\
\hdashline K_{2} \hat{M}_{p}^{-1}
\end{array}\right)\right\| \leq \max _{i}\left(k_{1 i}, k_{2 i}\right)
$$

and

$$
\begin{aligned}
& Q=\left(\begin{array}{c:c}
K_{1}^{2} & \Theta \\
\hdashline \Theta & K_{2}^{2}-K_{1}
\end{array}\right)=\left(\begin{array}{c:c}
\operatorname{diag}\left(k_{1 i}^{2}\right) & \Theta \\
\hdashline \Theta & \operatorname{diag}\left(k_{2 i}^{2}-k_{1 i}\right)
\end{array}\right) \\
& \lambda_{\min }(Q)=\min _{i}\left(k_{1 i}^{2}, k_{2 i}^{2}-k_{1 i}\right)
\end{aligned}
$$

where $\hat{D}_{p}$ is the short expression of the matrix $\hat{M}_{p}(\underline{\alpha}, \underline{\theta})=\left(d_{i j}(\underline{\alpha}, \underline{\theta})\right)$ and

$$
\gamma=\left\|\hat{M}_{p}^{-1}\right\|=\max _{1 \leq i \leq 2} \sum_{j=1}^{2}\left|d_{i j}(\underline{q}, \underline{\hat{\theta}})\right|
$$

Hence, it deduces:

$$
\begin{gathered}
\frac{\|P \Phi\| \delta}{\lambda_{\min }(Q)}=\frac{\gamma \delta \max _{i}(k, a k)}{\min _{i}\left(k^{2}, a^{2} k^{2}-k\right)}=\frac{\gamma \delta a k}{\min _{i}\left(k^{2}, a^{2} k^{2}-k\right)} \\
\leq \frac{\gamma \delta a k}{\min _{i}\left(k^{2},\left(a^{2}-1\right) k^{2}\right)}=\frac{\gamma \delta a}{k}
\end{gathered}
$$

and from which to find out:

$$
\lim _{k \rightarrow \infty} \frac{\gamma \delta a}{k}=0
$$

Therefore, by any given $\beta>0$ always exists a sufficiently large number $k>0$ such that:

$$
\Xi(\mathbb{Z}) \leq \frac{\gamma \delta a}{k}<\beta
$$

which affirms the rightness of Theorem 1 .

Finally, the desired rotational speed of tail and main rotor are calculated by following equations:

$$
\begin{gathered}
\omega_{h}^{*}= \begin{cases}\sqrt{\frac{\mathfrak{I}_{\text {proph }}}{k_{f h p} \times l_{t} \times \cos \alpha_{v}}} & \mathfrak{I}_{\text {proph }} \geq 0 \\
-\sqrt{\frac{-\mathfrak{I}_{\text {proph }}}{k_{f h n} \times l_{t} \times \cos \alpha_{v}}} & \mathfrak{I}_{\text {proph }}<0\end{cases} \\
\omega_{v^{*}}= \begin{cases}\sqrt{\frac{\mathfrak{I}_{\text {propv }}}{k_{f v p} \times l_{m}}} & \mathfrak{I}_{\text {prop }} \geq 0 \\
-\sqrt{\frac{-\mathfrak{I}_{\text {proph }}}{k_{f v n} \times l_{m}}} & \mathfrak{I}_{\text {prop }}<0\end{cases}
\end{gathered}
$$


From the equations (37) and (38), the input voltages of the tail motor and the main motor can be calculated by the inner control loop. This control loop, the PID controller is designed to give the input voltages $U_{h}, U_{v}$ applied to the two motors from the rotational speed errors. The structure of control system is described in the Fig 4.

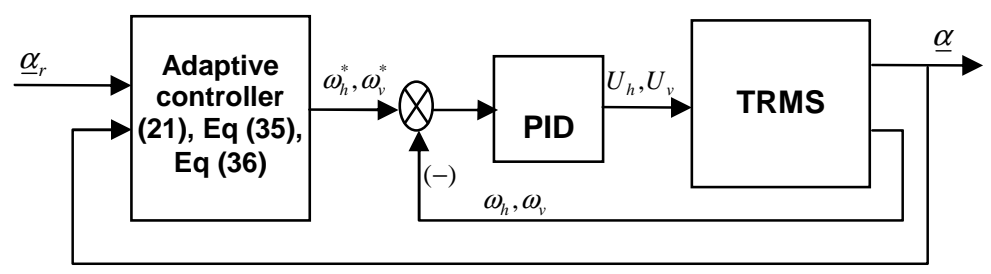

Figure 4. Structure of the closed loop system with two control loops: angle control loop and rotational speed loop.

\section{SIMULATION AND EXPERIMENTAL RESULTS}

In this part, we show the simulation and experimental results obtained by applying the adaptive controller (21) to TRMS with physical and defined parameters listed in the appendix. The simulation results are plotted in Figs 5-13, by using Matlab-Simulink R2007, in this simulation the friction torques of two channels are considered

$$
\begin{aligned}
& \tau_{\text {frich }}=\operatorname{sign}\left(\dot{\alpha}_{h}\right)\left(0.03 \times\left|\dot{\alpha}_{h}\right|+3 \times 10^{-4}\right)(\mathrm{Nm}) \\
& \tau_{\text {fricv }}=\operatorname{sign}\left(\dot{\alpha}_{v}\right)\left(0.0024 \times\left|\dot{\alpha}_{v}\right|+5.69 \times 10^{-4}\right) \quad(\mathrm{Nm})
\end{aligned}
$$

and the cable torque is

$$
\tau_{\text {cable }}=0.0016 \times\left(\alpha_{h}+0.0002\right)(N m)
$$
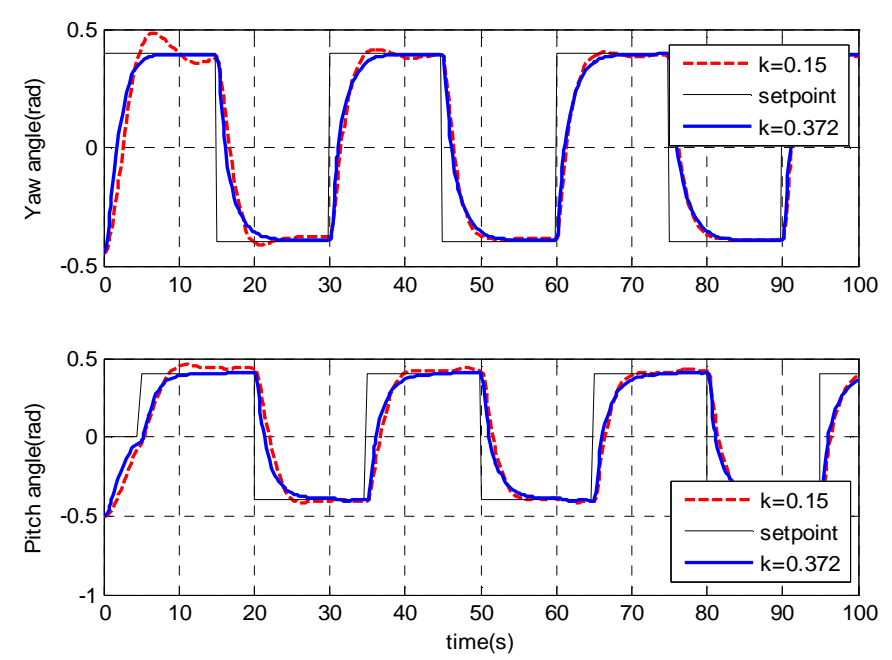

Figure 5. Simulation angle responses of yaw and pitch of TRMS controlled by adaptive controller (21) with $\mathrm{k}=0.15$ and $\mathrm{k}=0.372$. 

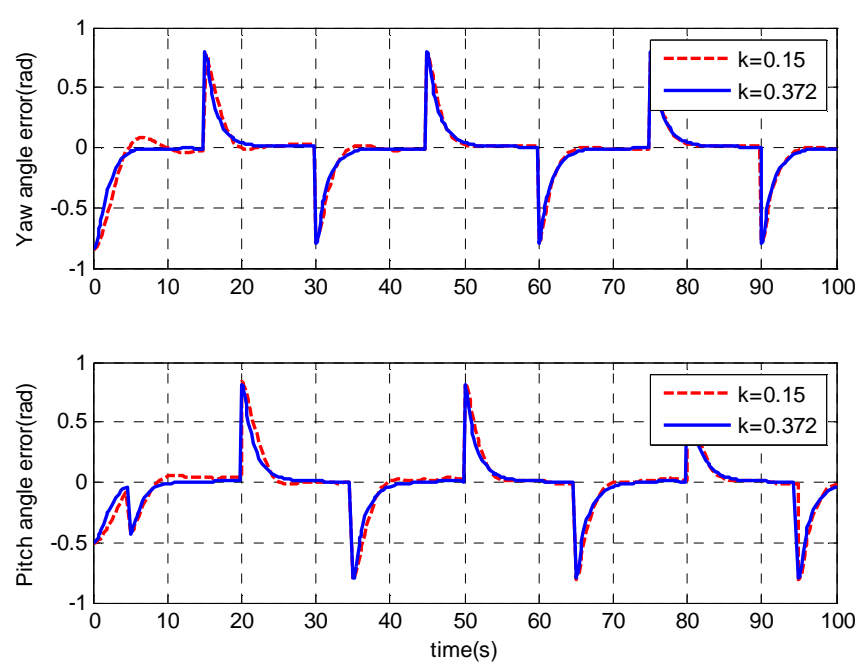

Figure 6. The errors of yaw and pitch angle with $\mathrm{k}=0.15$ and $\mathrm{k}=0.372$.

In Figs.5-10, there are the simulation results driven by adaptive controller (21) with $\mathrm{k}=$ 0.15 and $\mathrm{k}=0.372$ in the case no external disturbance acting on the TRMS, the system is only influenced by the uncertain parameters and the input disturbances.

The Fig.5 and Fig.6 represent the responses of the yaw and pitch angles tracking and tracking errors to the desired pulse signals (the hardest situation) whose level changes repeatedly between $-0.5 \mathrm{rad}$ and $0.5 \mathrm{rad}$ with period 30 seconds. After transient period of 5 seconds, the yaw and pitch angles track smoothly to the desired signals, with $\mathrm{k}=0.15$ and $\mathrm{k}=0.372$, maximum angle errors are $0.1 \mathrm{rad}$ and $0.05 \mathrm{rad}$, respectively.
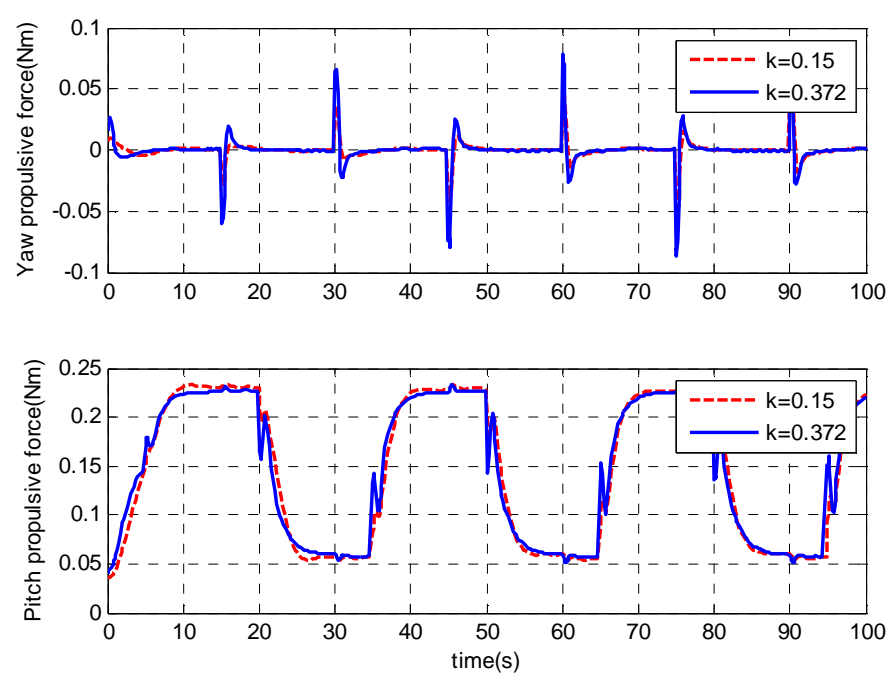

Figure 7. The yaw and pitch propulsive forces applied to the tail rotor and main rotor. 

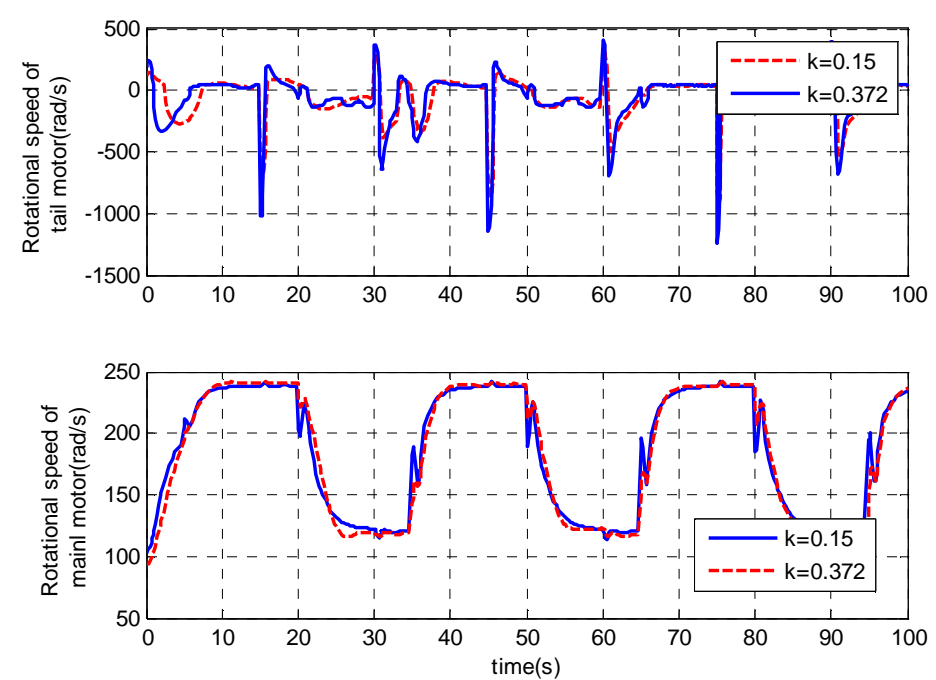

Figure 8. The rotational speeds of tail and main rotor.
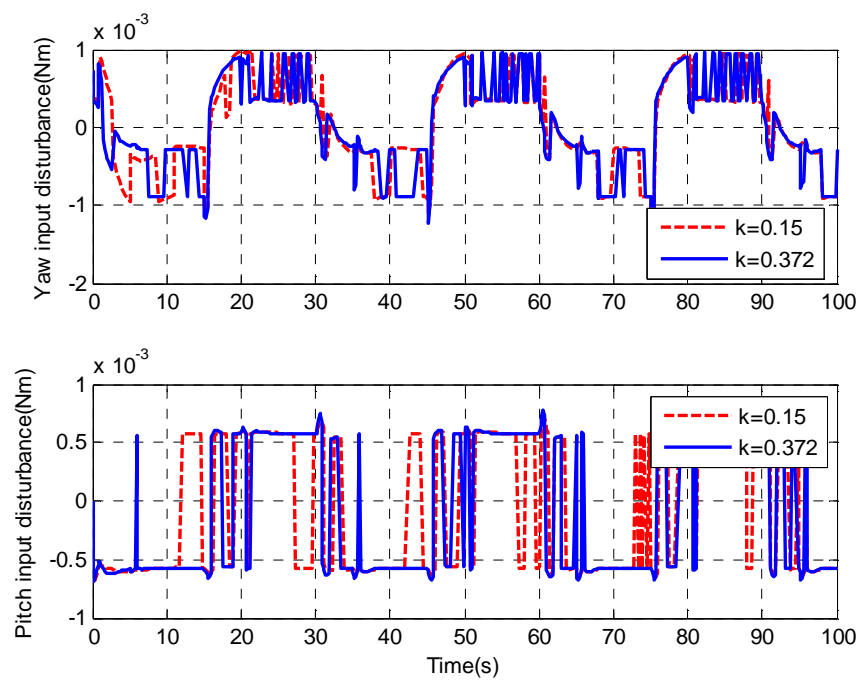

Figure 9. The yaw and pitch input disturbances.

The yaw propulsive force and the pitch propulsive force shown in the Fig. 7, the Fig. 8 refer to the rotational speed of tail motor and main motor, respectively. To track the desired signals at level changing times, the maximum rotational speed of the tail motor is approximately $1000 \mathrm{rad} / \mathrm{s}$ and of the main rotor is $250 \mathrm{rad} / \mathrm{s}$. The input disturbances caused by the friction force, the flat cable force, the effects of the speed of the main rotor on the horizontal movement and the speed of tail rotor to the vertical movements is depicted in the Fig. 9. The adaptive parameters of the controller are updated by the time as shown in the Fig. 10. 

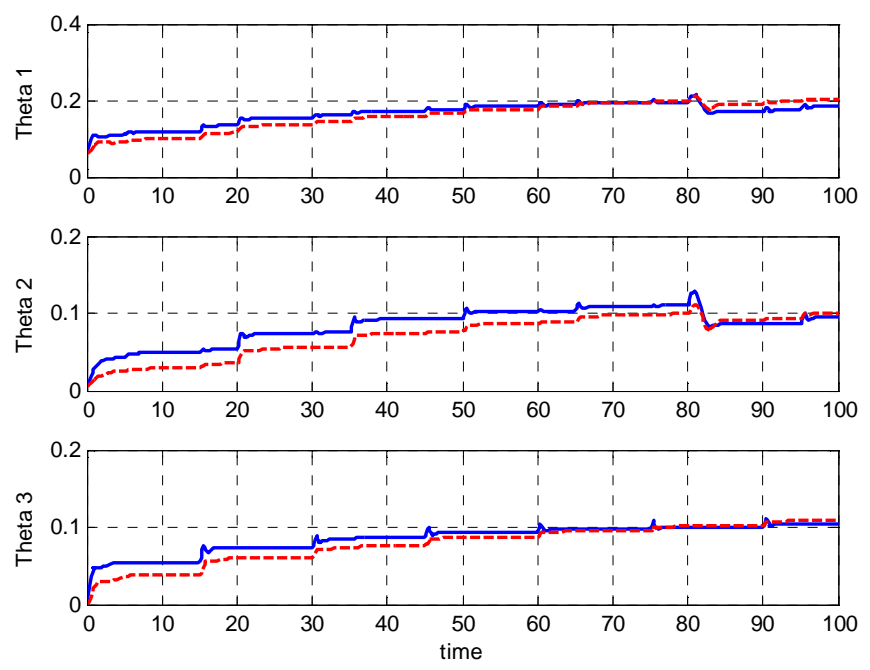

Figure 10. The adaptive parameters.

The Fig. 11 to the Fig. 13 show the response of the angles of TRMS attenuating the external disturbances acting on the yaw and the pitch angles at the time of 35 seconds and 80 seconds respectively.
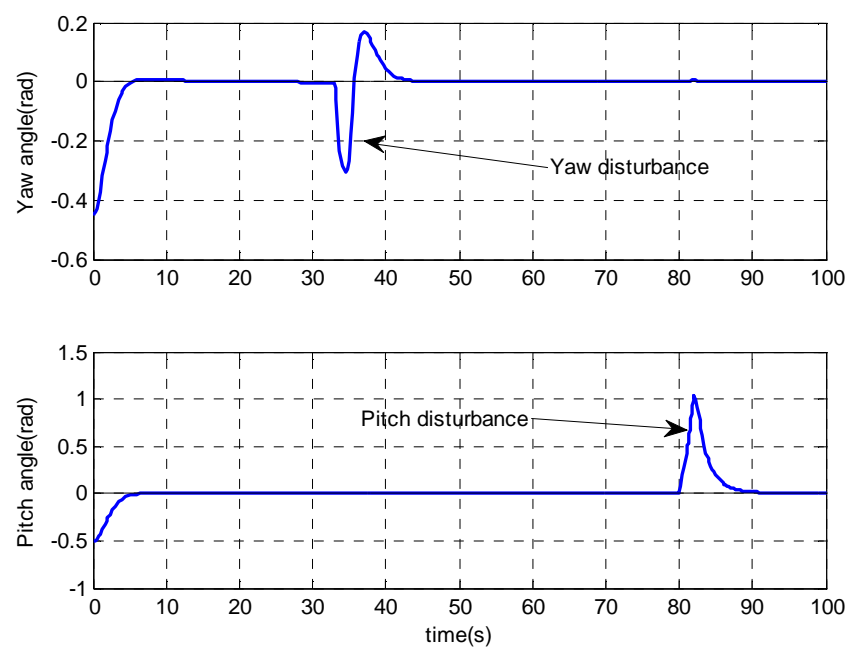

Figure 11. The responses of the angles with the external disturbance acting on the yaw and pitch. 

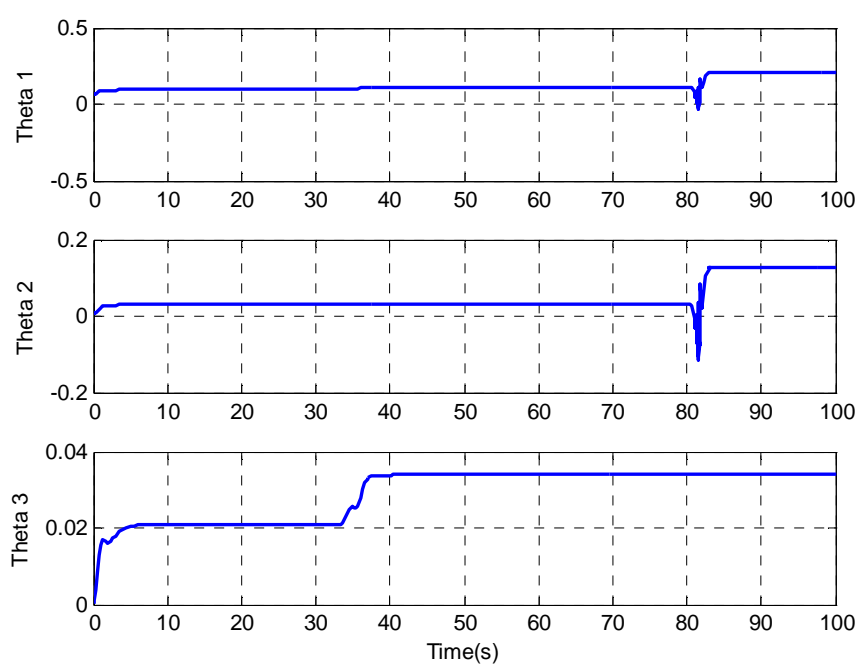

Figure 12. The varying of adaptive parameters.

Fig. 11 represents the responses of the yaw and pitch angles in the case the yaw and pitch acting to the TRMS, the yaw external disturbance can be attenuated with the overshoot in the response of the yaw while there is no overshoot in pitch response. Fig. 12 depicts the varying of the adaptive parameters, this parameters are adapted in the transient periods and the periods with external disturbance acting on the TRMS, in the stable state they are the constants.
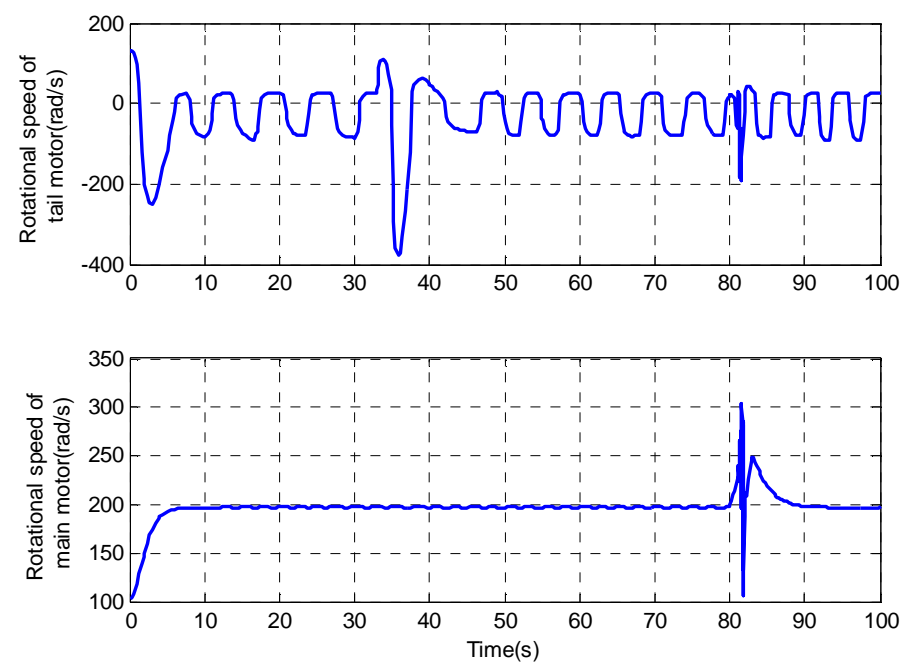

Figure 13. The rotational speeds of tail and main rotor in case the external disturbance acting.

To validate the performance of the controller, the experimental systems is depicted in Fig. 14 at the Instrument and Control Lab (310-TN, Electronics Faculty) of Thai Nguyen University of Technology (TNUT). To obtain the response of the TRMS we use the DSP 1103 PPC controller board supplied by dSPACE, control algorithm is installed in the computer with matlab/simulink R2007. After compiling, the control file is transferred to the DSP 1103 and angles of TRMS are monitored by Control Desk software. 


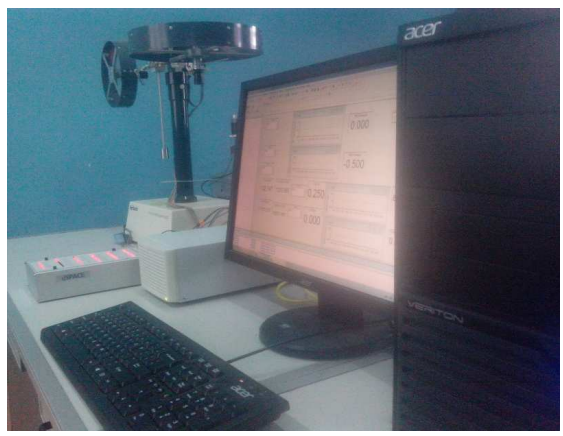

Figure 14. The setup of the experimental system,

Figure 15 refers to the experimental results, the yaw angle response tracks to the desired yaw angle formed in $0.45 \mathrm{rad}$ step signal at 40 seconds with error $0.01 \mathrm{rad}(1 \%)$. The pitch angle is kept at $0 \mathrm{rad}$, there are high peaks at the times the yaw angle changing suddenly, so after transient period the pitch angle error is about 0.01rad. Comparing with the methods in [4],[7],[9] and [17] performance of closed loop system is better. In the future works, we modify the ways to implement this controller in practice in order to reduce the angle errors by choosing appropriately the signal filters, simplifying the control algorithm and calibrating the input/output signals.
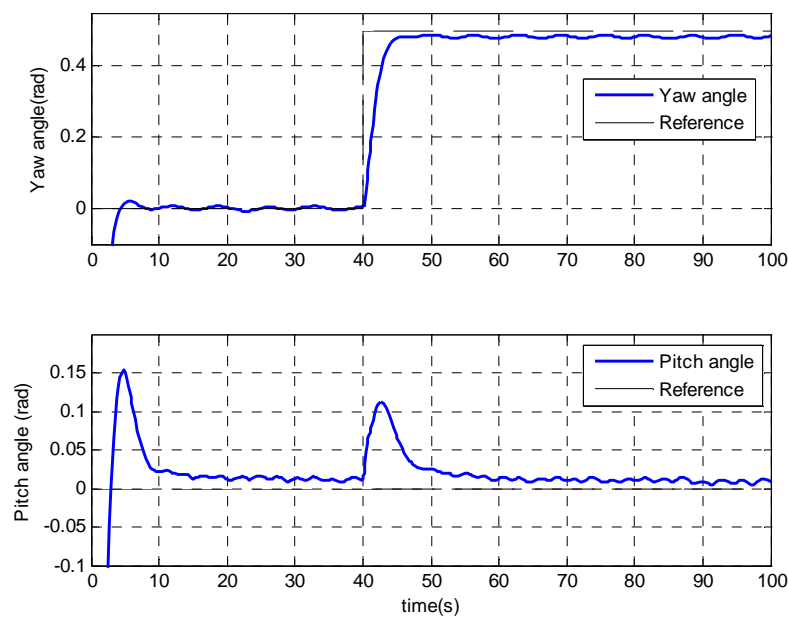

Figure 15. The closed loop step responses of yaw and pitch angles.

\section{CONCLUSION}

This paper introduces the adaptive controller design based on the ISS stabilization to angle track problem for TRMS. In order to design the controller, the mathematical model of TRMS is rewritten in Euler-Lagrange forced model with uncertain parameters and input disturbance. By conssidering carefully the model, we found that the energies depended on the mass of TRMS'parts are uncertain parameters, the flat cable force, the effects of the speed of the main rotor on the horizontal movement and the speed of tail rotor to the vertical movements are the input disturbances acting on the inputs of TRMS. The adaptive controller is designed based on the ISS stabilization with the bounded input disturbances. By choosing appropriately adaptive 
controller parameters, the effects of the input disturbances to the yaw and pitch angles will be attenuated. The robustness of closed loop with uncertain parameters and input disturbances is shown by proofing the proposed theorem together with the simulation and experimental results.

\section{APPENDIX}

The physical parameters supplied by the Feedback Instruments Limited and defined parameters of TRMS.

Table 1. The physical parameters supplied by the Feedback Instrument.

\begin{tabular}{|c|c|c|c|c|c|}
\hline$m_{b}$ & $\begin{array}{l}\text { Mass of the counter- } \\
\text { weight beam }\end{array}$ & $0.022 \mathrm{~kg}$ & $k_{g}$ & Gyroscopic constant & 0.2 \\
\hline$m_{c b}$ & $\begin{array}{l}\text { Mass of the counter- } \\
\text { weight }\end{array}$ & $0.068 \mathrm{~kg}$ & $k_{m}$ & Positive constant & $2 \times 10^{-4}$ \\
\hline$m_{m}$ & $\begin{array}{l}\text { Mass of main part of } \\
\text { the beam }\end{array}$ & $0.014 \mathrm{~kg}$ & $k_{t}$ & Positive constant & $2.6 \times 10^{-5}$ \\
\hline$m_{m r}$ & $\begin{array}{l}\text { Mass of the main DC } \\
\text { motor }\end{array}$ & $0.236 \mathrm{~kg}$ & $l_{t}$ & $\begin{array}{l}\text { Length of tail part of } \\
\text { the beam }\end{array}$ & $0.282 \mathrm{~m}$ \\
\hline$m_{m s}$ & $\begin{array}{l}\text { Mass of the main } \\
\text { shield }\end{array}$ & $0.219 \mathrm{~kg}$ & $l_{m}$ & $\begin{array}{l}\text { Length of main part of } \\
\text { the beam }\end{array}$ & $0.246 \mathrm{~m}$ \\
\hline$m_{t}$ & $\begin{array}{l}\text { Mass of the tail part of } \\
\text { the beam }\end{array}$ & $0.015 \mathrm{~kg}$ & $l_{b}$ & $\begin{array}{l}\text { Length of counter- } \\
\text { weight beam }\end{array}$ & $0.29 \mathrm{~m}$ \\
\hline$m_{t r}$ & $\begin{array}{l}\text { Mass of the tail DC } \\
\text { motor }\end{array}$ & $0.221 \mathrm{~kg}$ & $l_{c b}$ & $\begin{array}{l}\text { Distance between } \\
\text { counterweight and joint }\end{array}$ & $0.276 \mathrm{~m}$ \\
\hline$m_{t s}$ & Mass of the tail shield & $0.119 \mathrm{~kg}$ & $k_{f h p}$ & Positive constant & $1.84 \times 10^{-6}$ \\
\hline$r_{m s}$ & $\begin{array}{l}\text { Radius of the main } \\
\text { shield }\end{array}$ & $0.155 \mathrm{~m}$ & $k_{f h n}$ & Positive constant & $2.2 \times 10^{-7}$ \\
\hline$r_{t s}$ & $\begin{array}{l}\text { Radius of the tail } \\
\text { shield }\end{array}$ & $0.1 \mathrm{~m}$ & $k_{f v p}$ & Positive constant & $1.62 \times 10^{-5}$ \\
\hline$h$ & $\begin{array}{l}\text { Length of the offset } \\
\text { between base and joint }\end{array}$ & $0.06 \mathrm{~m}$ & $k_{f v n}$ & Positive constant & $1.08 \times 10^{-5}$ \\
\hline$g$ & $\begin{array}{l}\text { Gravitational } \\
\text { acceleration }\end{array}$ & $9.8 \mathrm{~m} / \mathrm{s}^{2}$ & & & \\
\hline
\end{tabular}

The defined parameters of TRMS model:

$$
\begin{aligned}
& \begin{array}{l}
m_{T_{1}}=m_{t}+m_{t r}+m_{t s}+m_{m}+m_{m r}+m_{m s}, l_{T_{1}}=\frac{\left(0.5 m_{m}+m_{m r}+m_{m s}\right) l_{m}-\left(0.5 m_{t}+m_{t r}+m_{t s}\right) l_{t}}{m_{T_{1}}} \\
m_{T_{2}}=m_{b}+m_{c b}
\end{array} \\
& l_{T_{2}}=\frac{0.5 m_{b} l_{b}+m_{c b} l_{c b}}{m_{T_{2}}}, J_{1}=\left(m_{t} / 3+m_{t r}+m_{t s}\right) l_{t}^{2}+\left(\frac{m_{m}}{3}+m_{m r}+m_{m s}\right) l_{m}^{2}+\frac{m_{m s}}{2} r_{m s}^{2}+m_{t s} r_{t r}^{2} \\
& J_{2}=\frac{m_{b}}{3} l_{b}^{2}+m_{c b} l_{c b}^{2}, J_{3}=\frac{m_{h}}{3} h^{2}
\end{aligned}
$$

\section{REFERENCES}

1. Feedback instruments Ltd, TRMS 33-949S User Manual, East Sussex, U. K (2005). 
2. Ahmad S.M., Chipperfield A.J., Tokhi, M.O. - Dynamic modelling and optimal control of a twin rotor MIMO system, Proceedings of IEEE National aerospace and electronics conference, Dayton, Ohio, USA (2000) pp. 391-398.

3. Pandey S.K. - Control of twin rotor MIMO system using PID controller with derivative filter coefficient, Electrical, Electronics and Computer Science, IEEE Students' Conference (2014) pp. 124-130.

4. Akash A.P, Prakash M., Hardik V. K. - Control of twin rotor MIMO system(TRMS) using PID Controller, National conference on emerging trends in computer, electrical \& electronics-ETCEE, International Journal of Advance Engineering and Research Development-IJAERD (2015) pp. 78-85.

5. Samir Zeghlache, Kamel Kara, Djamel Saigaa. -Type-2 fuzzy logic control of a 2-DOF helicopter (TRMS system), Central European Journal of Engineering 4 (3) (2014) pp 303-315.

6. Maryam J., Mohammad F. - Robust adaptive fuzzy control of twin rotor MIMO system, Methodologies and Application, Soft Computing Journal 17 (10) ( 2013) 1847-1860.

7. Sumit K.P. and Vijaya L. - Optimal Control of Twin Rotor MIMO System Using LQR Technique, Computational Intelligence in Data Mining 1 (2015) 11-21.

8. Anup K. E., Srinivasan A. and Mahindrakar D. - Terminal sliding mode control of a Twin rotor multiple-input multiple Output System, Preprints of the $18^{\text {th }}$ IFAC World Congress Milano, August 28 (2011) pp.10952-10957.

9. Rahideh A., Shaheed H.M and Bajodah A. H. - Adaptive non-linear model inversion control of a twin rotor multi-input multi-output system using artificial intelligence, Proc. IMechE Vol. 221 Part G: J. Aerospace Engineering (2007) pp.343-351.

10. Rahideh A., Shaheed H.M and Bajodah A. H. -Real time adaptive nonlinear model inversion control of a twin rotor MIMO system using neural networks, Engineering Applications of Artificial Intelligence 25 (6) (2012) 1289-1297.

11. Rahideh A., Shaheed H.M. - Mathematical dynamic modelling of a twin rotor multiple input-multiple output system, Proc. ImechE Vol. 221 Part I: J. Systems and Control Engineering (2007) pp. 89-101.

12. Sontag E. D. and Wang, Y. - On characterizations of the input to state stability property, System and Control Letters 24 (1995) 351-359.

13. Levis F.L., Dawson D.M. and Abdalla C.T. - Robot manipulator control, Theory and practice, Marcel Decker (2006).

14. Chi N.V., Phuoc N.D. - Adaptive ISS Stabilization Tracking Control Of Nonlinear Systems In The Presence Of Uncertainty And Input Noise With Guaranteed Tracking Errors, Journal of Computer Science and Cybernetics, VAST 29 (2) (2013) 132-141.

15. Chi N.V. and Phuoc N.D. - Adaptive tracking control based on disturbance attenuation and ISS stabilization of Euler-Lagrange nonlinear in the presence of uncertainty and input noise, Proceedings of IEEE 2th Int. Conference on Electrical Engineering and Automation Control (2011) 3698-3071.

16. Quan N.T. and Phuoc N.D. - Robust and adaptive control of Euler-Lagrange systems with an attractor independent of uncertainties, Proceedings of IEEE 12th Int. Conference on Control, Automation and Systems ICCAS (2012) 1309-1312.

17. Andrew E. P. - A Study of Advanced Modern Control Techniques Applied to a Twin Rotor MIMO System, Master of Science in Electrical Engineering, Department of Electrical and Microelectronic Engineering, Rochester Institute of Technology, Rochester, New York, (2014). 
